\title{
Ultrasonography of the infant hip
}

\section{Sorin M. Dudea}

Radiology Department, "Iuliu Hațieganu” University of Medicine and Pharmacy, Cluj-Napoca, Romania

Ultrasonography (US), as a diagnostic instrument, represented an unchallenged medical revolution in biliary disease but in cardiology, obstetrics and phlebology, as well. In the panoply of the golden applications of US, the use of the method for the early diagnosis and monitoring of developmental hip dysplasia was, somehow, unnoticed. Although available for more than 30 years and having a widely certified and thoroughly proven diagnostic value, hip US still struggles, in certain regions, with old fashioned radiography, to occupy its well deserved place.

The reasons of the slow and, at times, difficult coming into prominence of hip US are multiple.

A first explanation lies in the very different way of interpreting the importance of diagnostic criteria by the pediatric orthopedic surgeons. In a recent study [1] aimed at the members of this specialty in Europe, the authors concluded that only clinical assessment was a consistent agreement feature while the importance of patient history, US and radiography was rated inconsistently. Regional variations in the rating of the importance of diagnostic criteria were also observed.

Ignorance and distrust are other probable causes. This may be the likely explanation of the fact that there still are, in Europe, regions where the family practitioner continues to request clinical and radiographic exam at

Received Accepted

Med Ultrason

2013, Vol. 15, No 4, 259-260

Corresponding author: Prof. Dr. Sorin M. Dudea Radiology Department Emergency Clinical University Hospital "Iuliu Haţieganu" University of Medicine and Pharmacy, Cluj-Napoca 1-3 Clinicilor str 400006, Cluj-Napoca, Romania E-mail: dudea@clicknet.ro the baby's age of 4-5 months and the pediatric orthopedic surgeon chooses to rest his clinical judgment and treatment options upon these information. In the lack of enforcement or coherent national or regional programs, the use of US may be sporadic, finding itself at the mercy of the orthopedic surgeon's understanding and acceptance.

The history of hip US is marked by slow acceptance and initial denial. Fifteen years passed between the moment of the original description of the method and its wide scale application in countries like Germany or Switzerland, in the 90's.

The difficult acceptance of the method was often due to the misunderstanding of the technique or to hasty attempts to come up with not always justified changes.

One major incrimination of the method, often repeated in the early days, was its operator dependence and uncertain reproducibility. In fact, these drawbacks arise just from misunderstanding and non-compliance with the quality criteria assumed by the technique. The efforts to standardize and "automate" the method, deployed by those who initially described it, changed radically today's practice of hip US.

The paper of Professor Reinhard Graf [2] - the father of the method - published in the current issue of the journal, brings about a welcome remembrance on the existence and implications of hip US and offers an authoritative overview on the sources of error and ways to avoid them.

Just as in any other application, the method is only as good as the operator is, therefore the continuous assessment of the quality of the medical act is of paramount importance. To keep seeing, in 2013, radiographs with developmental hip dysplasia diagnosed at the baby's age of 5 to 7 months is, simply, bad medicine. 
The virtue of the paper in this issue is to remember us, in the era of contrast and elastography, that next to biliary, heart, obstetric and venous disease, US has changed forever the face of medicine in the matter of early diagnosis and therapeutic surveillance of hip developmental dysplasia.

\section{References}

1. Roposch A, Liu LQ, Protopapa E. Variations in the use of diagnostic criteria for developmental dysplasia of the hip. Clin Orthop Relat Res 2013; 47: 1946-1954.

2. Graf R, Mohajer M, Plattner F - Hip sonography update. Quality-management, catastrophes - tips and tricks. Med Ultrason 2013; 4: 266-303. 